\title{
AN OPTIMAL IMAGING-SCHEDULING ALGORITHM FOR THE MULTI-STRIP IMAGING-MODE OF THE HIGH-RESOLUTION AGILE SATELLITES BASED ON CERTAIN STEP-SIZE SEARCH
}

\author{
Bo Yang, Mi Wang, Shuying Jin, Jun Pan \\ State Key Laboratory of Information Engineering in Surveying, Mapping and Remote Sensing, Wuhan University, \\ China-93341186@qq.com, wangmi@1mars.whu.edu.cn,jsy@346936594@qq.com,hero_pj@sina.com
}

\section{Commission I, WG I/6}

KEY WORDS: high resolution; agile satellite; multi-strip imaging-mode; imaging-schedule

\begin{abstract}
:
The high flexibility of the agile satellite can significantly improve its ability to obtain data. Compared with ordinary satellite platforms, the imaging-scheduling is more important, and is the basis to ensure the efficiency and quality of data for the agile satellite. To be aimed at the multi-strip imaging-mode of the agile satellite, from the perspective of the image-quality, a imagingscheduling algorithm based on certain step-size search in the multi-strip imaging-mode of the agile satellite is proposed, and the influence of the selection of the imaging time-window on the multi-strip image quality is analyzed quantitatively through the experimental data. The results show that the optimal image data can be obtained through the image-scheduling algorithm.
\end{abstract}

\section{INTRODUCTION}

With the continuous development of space technology, Europe and the United States and other aerospace-powers have started the development of high-resolution agile satellite platform. For example, the IKONOS-2 satellite launched by the United States in September 1999 has the agile observation capabilities of nadir-view, forward-view, backward-view and side-view. WorldView-1 launched in September 2007 has been the most flexible one of the on-orbit Earth-observation satellites so far. In addition, French PLEIADES Constellation launched in2008, Russia Topsat launched at the end of 2005 and so on, are both agile satellites (Chen et al., 2011). Compared with ordinary remote-sensing satellite platform, high-resolution agile satellite is of the high freedom in more than one direction, has the ability to rapidly change attitude and precisely control attitude along the three direction of pitch, roll and yaw. These performances make the agile satellites can acquire the images of the target area in the ground with different attitudes. Therefore, agile satellite with higher flexibility and rapid pointing ability, has become an important development direction in the field of the satellite technology. Agile satellite with flexible and agile attitude maneuver capability can improve the satellite application performance, achieve large-range and multi-stripe observation, solve the contradiction between the "high resolution" and "wide-swath", and provides a good way in the high-resolution remote sensing image applications.

Multi-strip imaging is one kind of data acquisition mode of the high-resolution agile satellite. Through changing imaging attitude, the agile satellite can acquire multi-strip images in a transit, achieve imaging coverage of the large-range target-area, and so improves the efficiency of data-acquisition.Figure 1 shows the multi-strip imaging mode of the agile satellite. When acquiring the multi-strip images of the target area, the high agility makes the agile satellite can choose different imaging time-window. There is great difference among the quality of the images obtained in different imaging time-windows. To be aimed at the multi-strip imaging-mode of the agile satellite, from the perspective of the subsequent processing and application, this paper establishes a multi-strip image quality evaluation model, researches imaging scheduling algorithm, makes the decision of the optimal imagingparameters(including the time-windows and imaging attitudes), so that the multi-strip images is of the highest quality.

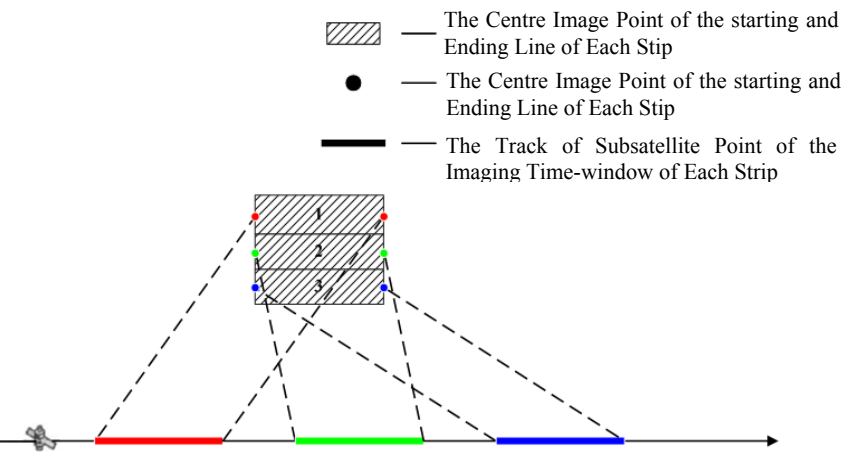

Figure 1. The agile satellite multi-strip imaging-mode

To be aimed at the satellite imaging scheduling, domestic and foreign scholars have conducted a lot of research for a variety of satellite and application requirements, and varied imaging scheduling models and optimization algorithms are proposed, such as ant colony algorithm, greedy algorithm, the local optimal search algorithm, dynamic programming algorithm, constraint programming algorithm and so on (Lemaitre et al., 2002; Aarts and Lenstra, 2003; Bensana et al., 1996; Vasquez and Hao, 2001). The main purpose of these algorithms is to obtain the image of maximum-coverage with minimal resource consumption under various constraints (Lin et al., 2005; Gabrel and Murat, 2003; Bai et al., 2010; He 2004), and the image quality is not taken as the main factors to be considered. From the perspective of image quality, the agile satellites acquiring the multi-strip images of single target area, this paper establishes the image quality evaluation model and the objective function, make research of scheduling algorithm, make decision of the imaging time-window and attitude parameter of each strip; Meanwhile, the improvement of the 
image quality by scheduling is analyzed quantitatively through experimental data.

\section{THE ESTABLISHMENT OF THE EVALUATION MODEL OF THE QUALITY OF MULTI-STRIP IMAGES}

Radiation differences and geometric resolution differences among strip images of agile satellite are the main factors to affect the subsequent processing and applications. The smaller difference is more conducive to bring the potential of the multistrip images data into full play. Based on that, this article establishes the evaluation model from these two aspects, make analysis of quality of multi-strip images.

\subsection{The Indicator of the Difference of Geometric Resolution}

Assume that the geometric resolution of each strip image is $S_{i}$ ( $i=1,2,3 \ldots, n$ ), the difference index $\eta$ of geometric resolution among each strip image is calculated according to equation (1), where $\bar{S}$ is the mean value of each strip image geometric resolution.

$$
\eta=\frac{\sum_{i=1}^{n} \frac{\left(S_{i}-\bar{S}\right)^{2}}{n}}{\bar{S}} \quad \bar{S}=\frac{\sum_{i=1}^{n} S_{i}}{n}
$$

\subsection{The Indicator of the Difference of Radiation}

For a place on Earth, the solar altitude angle is the angle between the sunlight incident direction and the ground plane. The solar altitude angle is the most important factor to determine the solar thermal energy which the Earth's surface receive, and therefore also an important factor to the radiance of a scene of image. In this paper, the solar elevation angle difference is taken as the evaluation indicator of the radiation difference among each strip image.

The solar altitude angle $H_{s}$ is numerically equal to the altitude of the sun in the horizon coordinate system, and varies with the local time and the solar declination. Suppose $\delta$ is the solar declination, $\varphi$ and $\tau$ are the geographic latitude and local time of the observation place respectively, then the solar altitude angle $H_{s}$ can be calculated via equation(2)(Li et al., 1993)

$$
\sin H_{s}=\sin \varphi \cdot \sin \delta+\sin \varphi \cdot \cos \delta \cdot \cos \tau
$$

The specific calculation model of the solar declination and local time can be referenced to the related literature.

After calculating the solar altitude angle $H_{s i}$ ( $i=1,2,3 \ldots, n$ ) of each strip image, the evaluation indicator of the radiation difference $\phi$ can be calculated according to equation (3).

$$
\phi=\frac{\sum_{i=1}^{n} \frac{\left(H_{s i}-\overline{H_{s}}\right)^{2}}{n}}{\overline{H_{s}}} \quad \overline{H_{s}}=\frac{\sum_{i=1}^{n} H_{s i}}{n}
$$

\subsection{The Evaluation Model of the Quality of Multi-Strip Images}

According to the aforementioned geometric resolution difference indicator and radiation difference indicator, this paper build the evaluation model of the quality of the multistrip images, as shown in equation (4).

$$
\mu=\frac{1}{W_{\eta} \cdot \eta+W_{\phi} \cdot \phi}=\max
$$

Where, $W_{\eta}$ and $W_{\phi}$ are the weight of geometric resolution difference and radiation difference respectively, according to the need to set.

\section{THE ALGORITHM OF MULTI-STRIP IMAGING SCHEDULING OF THE AGILE SATELLITE}

Satellite imaging scheduling, is that, under certain constraints, imaging parameters is determined by the algorithm, so that the value of the objective function is optimal (Galvao et al., 2000). In this paper, the objective function is the evaluation model of the quality of the multi-strip images, and the constraints are only that imaging attitudes is required to meet the angle constraints due to the mobility restrictions: $\varphi \leq \varphi_{\text {MAX }}$ (where $\varphi$ and $\varphi_{\text {MAX }}$ are imaging cone angle and maximum imaging cone angle respectively).Therefore, the imaging scheduling mathematical model is shown as equation(5).

$$
\left\{\begin{array}{l}
\mu=\max \\
\varphi_{i} \leq \varphi_{M A X}
\end{array}(i=1,2 \ldots, n)\right.
$$

If each strip of the target area and its starting imaging time $T_{s i}$ ( $i=1,2 \ldots, n$ ) are determined, its imaging attitude and ending imaging time $T_{e i}(i=1,2 \ldots, n)$ can be determined. In addition, the relation between the starting imaging time $T_{s(i+1)}$ of the strip $\mathrm{Si}+1$ and the ending imaging time $T_{e i}$ of the strip Si satisfies the equation: $T_{S(i+1)}=T_{e i}+T_{A}$ (where $T_{A}$ represents the attitude-adjust time).Based on the above analysis, it can be inferred that if the starting imaging time $T_{s 1}$ of the first strip image, the imaging time-window $\left(T_{s i}, T_{e i}\right)$ and imaging attitude angle $\left(\right.$ pitch $\left._{i}, \operatorname{roll}_{i}, \operatorname{yaw}_{i}\right)(i=1,2 \ldots, n)$ of each strip image can be calculated. Therefore, the key point of the imaging scheduling of the multi-strip mode of the agile satellites is to determine the optimal starting imaging time $T_{s 1}$ 
of the first strip. On the basis of the analysis and summary of the existing algorithms, this paper determines the optimal starting imaging time $T_{s 1}$ of the first strip based on the stepsize search strategy (M. A. and K., 1968), and then calculates the imaging time-window and attitude of each strip. Assume that the strip number is $n$, this algorithm progressively search $T_{s 1}^{k}(k=1,2, \ldots m \ldots)$ for the optimal starting imaging time $T_{s 1}$ with a certain time-step $\Delta T$ in the visible time window $V T W_{1}\left(V T W S_{1}, V T W E_{1}\right)$ of the first strip, where $T_{s 1}^{k}$ meets the equation(6).For each $T_{s 1}^{k}(k=1,2, \ldots m \ldots)$, the imaging time-window $\left(T_{s i}, T_{e i}\right)^{k}$ and attitude (pitch $_{i}$, roll $_{i}$, yaw $\left._{i}\right)^{k}$ of each strip and the value $\mu^{k}$ of the image quality evaluation function are calculated, and then the $T_{s 1}^{k}$ which meets the equation(5) is considered as the optimal starting imaging time of the first strip, and the corresponding imaging time-window and attitude of each strip is the optimal imaging parameter via scheduling.

$$
\left\{\begin{array}{l}
T_{s 1}^{k}=V T W S_{1}+k \times D T \\
T_{s 1}^{k}<V T W E_{1}
\end{array} \quad(k=1,2, \ldots m \ldots)\right.
$$

The specific technology route is shown as figure 2 .

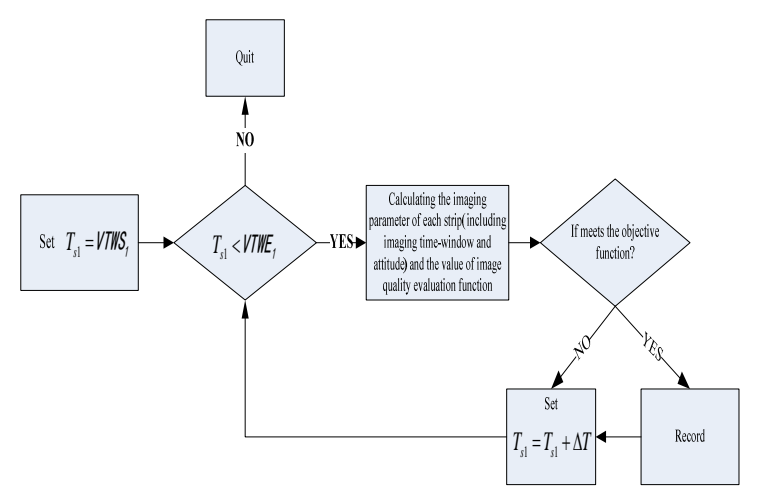

Figure 2. The technical route of optimal imaging decision

\subsection{Polynomial Fitting of the Satellite Exterior Orientation} Linear Element

Due to the influence of a variety of non-gravity, the actual orbit deviates from a Keplerian orbit. These non-gravity is called perturbation. Therefore, normally, the remote sensing satellite orbit is a smooth track.

Taking into account the smooth running of the satellite orbit, in a short period of time, the polynomial can be used to describe the remote sensing satellite's orbit, thus avoiding the complicated satellite stress analysis (Zhang, 2005.).

\subsection{The Calculation of the Imaging Attitude Parameter of Each Strip}

Not considering the install angles of the sensor, attitude parameters of the sensor, (pitch, roll, yaw) usually indicate the relationship between the body coordinate system and the orbital coordinate system. As the yaw angle is a fixed value, the attitude parameter to calculate is pitch and roll . As shown in figure 3, the vector $\mathrm{V}$ represents the principal optical axis; $\mathrm{XG} 、 \mathrm{YG}$ and $\mathrm{ZG}$ represent the three components of the body coordinate system.

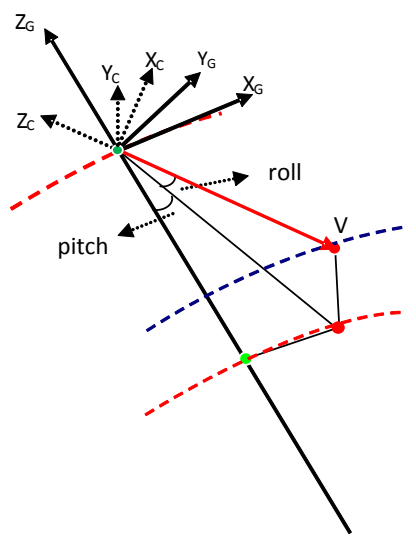

Figure 3. The calculation of the attitude parameter of the sensor

The centre image-point of the starting line, the corresponding ground point and project centre meet the collinear equation (7) (Zhang and Pan, 2003).

$$
\left[\begin{array}{c}
x \\
y \\
f
\end{array}\right]_{\text {cam }}=l R_{C G} R_{G F}\left(\left[\begin{array}{c}
X_{g} \\
Y_{g} \\
Z_{g}
\end{array}\right]-\left[\begin{array}{c}
X_{s} \\
Y_{s} \\
Z_{s}
\end{array}\right]\right)_{C T S}
$$

where, $R_{C G}$ and $R_{G F}$ represent the rotate matrix from the orbital coordinate system to the sensor coordinate system and from the Earth fixed coordinate system to the orbital coordinate system respectively; $(x, y, f)$ is the coordinate of the centre image-point of each strip image in the sensor coordinate system.

Matrix $R_{G F}$ is calculated via equation (8).

$$
\begin{aligned}
& R_{F G}=\left[\begin{array}{lll}
\left(X_{2}\right)_{X} & \left(Y_{2}\right)_{X} & \left(Z_{2}\right)_{X} \\
\left(X_{2}\right)_{Y} & \left(Y_{2}\right)_{Y} & \left(Z_{2}\right)_{Y} \\
\left(X_{2}\right)_{Z} & \left(Y_{2}\right)_{Z} & \left(Z_{2}\right)_{Z}
\end{array}\right] \\
& \left.\vec{Z}_{2}=\frac{\vec{P}(t)}{\|\vec{P}(t)\|}, \vec{X}_{2}=\frac{\vec{V}(t) L \vec{Z}_{2}}{\left\|\vec{V}(t) L \vec{Z}_{2}\right\|}, \vec{Y}_{2}=\vec{Z}_{2} L \vec{X}_{2}\right\} \\
& \vec{P}(t)=\left[\begin{array}{lll}
X_{s} & Y_{s} & Z_{s}
\end{array}\right]^{T} \\
& V(t)=\left[\begin{array}{lll}
X v_{s} & Y v_{s} & Z v_{s}
\end{array}\right]^{T}
\end{aligned}
$$

Where, $\left(X_{s}, Y_{s}, Z_{s}\right)$ and $\left(X_{v_{s}}, Y_{v_{s}}, Z_{v_{s}}\right)$ are the position and velocity of the centre of the satellite in the Earth fixed coordinate system; $R_{F G}$ represent the rotate matrix from the orbital coordinate system to the Earth fixed coordinate system. Assume the centre of a scan line image of the satellite sensor corresponds to the principal point of photograph, that is the 
imaging light and the principal optical axis coincide, Meanwhile, the imaging time of the starting line and the coordinate of the corresponding ground point of its centre image point in the Earth fixed coordinate system is given, the coordinate of the projection centre in the Earth fixed coordinate system can be interpolated through the orbit polynomial, and the coordinate of the principal optical axis $V$ in the orbital coordinate system, $\left(V_{X}, V_{Y}, V_{Z}\right)$ can be calculated via equation(9).

$$
\left[\begin{array}{c}
V_{X} \\
V_{Y} \\
V_{Z}
\end{array}\right]_{\text {轨 }}=R_{G F}\left(\left[\begin{array}{c}
X_{g} \\
Y_{g} \\
Z_{g}
\end{array}\right]-\left[\begin{array}{c}
X_{s} \\
Y_{s} \\
Z_{s}
\end{array}\right]\right)_{C T S}
$$

After calculating $\left(V_{X}, V_{Y}, V_{Z}\right)$, the coordinate of the principal optical axis $V$ in the orbital coordinate system, the attitude angles, pitch, roll and the cone angle $\varphi$ are calculated through equation(10).

$$
\left.\begin{array}{l}
\text { pitch }=\arctan \left(\frac{Y}{Z}\right) \\
\text { roll }=\arctan \left(\frac{X}{\sqrt{Y^{2}+Z^{2}}}\right) \\
\varphi=\arccos \mid \cos (\text { pitch }) \cos (\text { roll }) \mid
\end{array}\right\}
$$

If $\varphi>\varphi_{\max }\left(\varphi_{\max }\right.$ symbolizes the maximum cone angle of the satellite), the imaging scheduling scheme is not feasible.

\subsection{The Calculation of the Imaging Time-window of Each Strip}

If the starting imaging time $T_{s}$ is determined, the starting imaging time of the first strip $T_{s 1}$ equates to $T_{s}$. Simply calculating the ending imaging time of each strip $T_{e i}$, the starting imaging time of the next one is equal to $T_{e i}+T_{a}\left(T_{a}\right.$ is the attitude adjust time).Therefore, calculating the ending imaging time of each strip, $T_{e i}$, is key to calculate the imaging time-window of each strip $\left[T_{s i}, T_{e i}\right]$. The ending imaging time of each strip, $T_{e i}$, can be calculated according to equation(11).

$$
T_{e}=\frac{S}{v}
$$

Where, $S$ is the length of the strip, and $V$ symbolizes the velocity of satellite.

\section{EXPERIMENT AND RESULT ANALYSIS}

Based on the imaging scheduling algorithm for the multi-strip imaging mode of the agile satellite proposed by this paper, a program is written in $\mathrm{c}++$ language. The input parameters which the program needs include: the longitude and latitude coordinates of the four corners of the target area, satellite transit ephemeris data as well as the system parameters of the satellite sensor platform; Its output is a scheduling result file, including the strip number, imaging sequence, imaging time-window and attitude of each strip, geometric difference indicator, radiation difference indicator and the image quality evaluation indicator. The system parameters of the satellite platform in the experiment in this paper are listed in Table 1, and the target area is located in the Beijing, its range is approximately $40 \mathrm{~km}$ along the scan direction and $37 \mathrm{~km}$ across the scan direction.

Tab.1 The parameter of the agile-satellite platform and the sensor

\begin{tabular}{cl}
\hline Orbital Height & $650 \mathrm{~km}$ \\
\hline Principal distance & $10 \mathrm{~m}$ \\
\hline Detector size & $10 \mu \mathrm{m}$ \\
\hline Attitude adjust time & $10-15$ second \\
\hline Maximum cone angle & 45 degree \\
\hline Strip swath & $10 \mathrm{~km}$ \\
\hline
\end{tabular}

\begin{tabular}{|c|c|c|c|c|}
\hline $\begin{array}{l}\text { Strip } \\
\text { NO. }\end{array}$ & $\begin{array}{c}\text { Pitch } \\
\text { (degree) }\end{array}$ & $\begin{array}{c}\text { Roll } \\
\text { (degree) }\end{array}$ & $\begin{array}{l}\text { Starting } \\
\text { maging time } \\
\text { (second) }\end{array}$ & $\begin{array}{l}\text { Ending } \\
\text { imaging } \\
\text { time } \\
\text { (second) }\end{array}$ \\
\hline 1 & 20.1971 & -4.2492 & 30 & 35.3622 \\
\hline 2 & 11.9388 & -3.8654 & 45.3622 & 50.7247 \\
\hline 3 & 2.65273 & -3.3468 & 60.7247 & 66.0872 \\
\hline 4 & -6.78476 & -2.7416 & 76.0872 & 81.4496 \\
\hline 5 & -15.8038 & -2.1970 & 91.4496 & 96.8119 \\
\hline $\begin{array}{l}\text { Strip } \\
\text { NO. }\end{array}$ & $\begin{array}{l}\text { Geometric } \\
\text { Resolution } \\
\quad(\mathrm{m})\end{array}$ & $\begin{array}{c}\text { Solar altitude } \\
\text { angle } \\
(\text { degree })\end{array}$ & $\begin{array}{c}\text { Image } \\
\text { quality } \\
\text { indicator }\end{array}$ & \\
\hline 1 & 0.694841 & 67.85221 & & \\
\hline 2 & 0.665251 & 67.95914 & & \\
\hline 3 & 0.650167 & 67.93895 & & \\
\hline 4 & 0.654428 & 67.91876 & & \\
\hline 5 & 0.677633 & 67.89858 & 92.0839 & \\
\hline
\end{tabular}

The results are shown in Table 2 (the time in this experiment is on the basis of the starting time VTWS $S_{1}$ of the visible timewindow $V T W_{1}\left(V T W S_{1}, V T W E_{1}\right)$ of the first strip).

Tab.3 The image-scheduling result of the target area

The relationship between the starting imaging time $T_{s 1}$ of the first strip and the image quality indicator of the multi strip images is shown as figure 4 . 


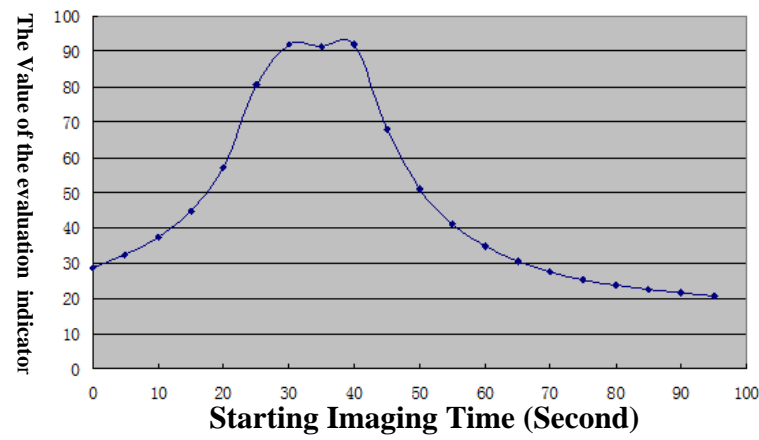

Figure 4. The relation between TS and the indicator of the image-quality

It is found via analyzing the above figures and tables that the choice of $T_{s 1}$ has great impact on the quality of the multi-strip images. As illustrated in figure $4, T_{s 1}$ equating to 0 second, the quality indicator is $28.8 ; T_{s 1}$ equating to 30 second, the quality indicator is $92.1 ; T_{s 1}$ equating to 90 second, the quality indicator is 21.52.In addition, the line gragh in figure 4 plot the relation between $T_{s 1}$ and the quality indicator of the multi-strip images, that the image quality is best when $T_{s 1}$ lies in the middle of the visible time-window of the first strip, and on the contrary is worse when lies in the beginning or end of the visible time-window of the first strip.

\section{CONCLUSION}

Agile satellite has the good characteristics of rapidly changing attitude. It can acquire the image of a wide range of target area by multi-strip imaging mode, as greatly improves the efficiency of data acquisition. To be aimed at the multi-strip imagingmode of the agile satellite, from the perspective of the imagequality, this paper proposed the imaging scheduling algorithm; meanwhile, the improvement of the image quality by scheduling is analyzed quantitatively through experimental data. Thus recommendation and guidance for the proper utilization of the resource of the agile satellites are proposed.

\section{ACKNOWLEDGEMENTS}

This paper is Supported by Program for New Century Excellent Talents in University, the National High Technology Research and Development Program (2012CB719902) and National Key Technology R\&D Program(2011AA120203).

\section{REFERENCES}

[1] Chen Yuning, Xing Lining, Chen Yingwu, 2011. Scheduling of Agile Satellite Based on Ant Colony Algorithm. Science Technology and Engineering, 11(3), pp.484-502.

[2] Lemaitre M., Verfaillie G., Jouhaud F., et al, 2002.Selecting and scheduling observations of agile satellites.Aerospace Science and Technology, 6, pp.367-381.

[3] Aarts EHL, Lenstra JK, 2003. Local Search in Combinatorial Optimization. Princeton University Press.
[4] Bensana E.,Verfaillie G.,Bataillie N.,et al,1996. Exact and approximate methods for the daily management of an earth observing satellite.Proceedings of SpaceOPS, Germany.

[5] Vasquez M., Hao J K,2001.A"Logic-Constrained" Knapsack Formulation and a Tabu Algorithm for the Daily Photograph Scheduling of An Earth Observation Satellite.Computational Optimization and Applications,20(2), pp.137-157.

[6] Gabrel V.,Vanderpooten D.,2002.Enumeration and interactive selection of efficient paths in a multiple criteria graph for scheduling an earth observing satellite[J].European Journal of Operational Research,139(3), pp.533-542.

[7] Wang Yanjuan, Zhang Hui, 2008. Research on Imaging Reconnaissance Satellite Scheduling Based on Genetic Algorithm. Ordanance Industry Automation, 27(10), pp.83-85.

[8] Lin W C, Liao D Y, Liu C Y, et al,2005.Daily imaging scheduling of an earth observation satellite[J].IEEE Transactions on Systems, Man and Cybernetics Part A, Systems and Humans ,35(2),pp.213-223.

[9] Gabrel V, Murat C, 2003. Mathematical Programming for Earth Observation Satellite Mission Planning. Operations Research in Space and Air.

[10] Bai Baocun, Xu Yifan, He Renjie, Chen Yingwu, 2010. A Maximum Coverage Model and Algorithm for Satellite Scheduling with Task Merging. Journal of Systems Engineering, 25(5), pp.651-658.

[11] He Renjie, 2004. Research on Imaging Reconnaissance Satellite Scheduling Problem. Changsha: Nation University of Defence Technology.

[12] Wang Haibo, Xu Minqiang, Wang Rixin, Li Yuqing, 2010. Gray Ant Colony System for Multi-objective Satellites Observation Scheduling. Journal of Harbin Institute of Technology, 42(11), pp.1686-1689.

[13] Xu Xueren, Gong Peng, Huang Xuezhi, Jin Yong, 2007. Study on Optimization Algorithms for Remote Sensing Data Collection Planning of Satellite. Journal of Remote Sensing, 2007, 11(1), pp.109-114.

[14] Lu Pan, Xu Peide, 2008. Scheduling of Imaging Reconnaissance Satellites (IRS) Based on Greedy Algorithm. Computer Simulation, 25(2), pp.37-40.

[15] Galvao R D, Espejo L G A, Boffey B, 2000. A comparison of Lagrangean and surrogate relaxations for the maximal covering location problem. European Journal of Operational Research, 24(1), pp.377-389.

[16] Li Xianhua, Huang Xueqiao, Chi Tianhe, Liu Yi, Wang Xiaoping, 1993. Computation of Solar Elevations and Azimuths at Pixels of Satellite Image. Acta Geodetica et Cartographica Sinica, 22(2), pp.149-154.

[17] M. A. Schumers, K. Steiglitz, 1968.Adaptive stepsize random search. IEEE Transactions on Automatic Control, 13, pp.270-276.

[18] Leonardo R. V., Hernan R, Jacob B, 2010.A robust variable step-size affine projection algorithm.Signal Processing, 90, pp.2806-2810.

[19] Zhang Guo, 2005. Rectification for High Resolution Remote Sensing Image under Lack of Ground Control Points. Wuhan: Wuhan University.

[20] Zhang Jianqing, Pan Li, 2003. Photogrammetry. Wuhan: Press of Wuhan University, pp.14-16. 